\title{
Dynamical instability in kicked Bose-Einstein condensates
}

\author{
J. Reslen, ${ }^{1}$ C. E. Creffield, ${ }^{1,2}$ and T. S. Monteiro ${ }^{1}$ \\ ${ }^{1}$ Department of Physics and Astronomy, University College London, Gower Street, London WC1E 6BT, United Kingdom \\ ${ }^{2}$ Departamento de Física de Materiales, Universidad Complutense de Madrid, E-28040, Madrid, Spain
}

(Received 30 July 2007; revised manuscript received 20 March 2008; published 18 April 2008)

\begin{abstract}
Bose-Einstein condensates subject to short pulses ("kicks") from standing waves of light represent a nonlinear analog of the well-known chaos paradigm, the quantum kicked rotor. Previous studies of the onset of dynamical instability (i.e., exponential proliferation of noncondensate particles) suggested that the transition to instability might be associated with a transition to chaos. Here we conclude instead that instability is due to resonant driving of Bogoliubov modes. We investigate the Bogoliubov spectrum for both the quantum kicked rotor (QKR) and a variant, the double kicked rotor (QKR-2). We present an analytical model, valid in the limit of weak impulses which correctly gives the scaling properties of the resonances and yields good agreement with mean-field numerics.
\end{abstract}

DOI: 10.1103/PhysRevA.77.043621

PACS number(s): 03.75.Lm, 05.45.-a, 03.65.Ta

\section{INTRODUCTION}

The production of Bose-Einstein condensates (BECs) in dilute atomic gases has opened up a new domain for research in quantum dynamics, since BECs are intrinsically phase coherent and can be controlled experimentally to an extremely high degree of precision [1]. An increasingly interesting aspect of the dynamics of BECs is that they represent a new arena for investigation of the interaction between nonlinearity and quantum dynamics, including quantum chaos [2-9].

A BEC subject to periodic short pulses, or kicks, from standing waves of light represents a nonlinear generalization of the well-known chaos paradigm, the quantum kicked rotor (QKR). The QKR has been realized using (noncondensed) cold atoms, permitting experimental investigation of a range of interesting chaos phenomena [10]. The regime where the kick period $T$ is a rational multiple of $\pi$ has also proved of particular interest: several studies have investigated the dynamics here with or without nonlinearity $[8,11,12]$. A number of experimental studies have also investigated kicked BECs [14]. However, these studies considered studied essentially the (linear) QKR resonances, and any nonlinearity, if present appears simply as a perturbation on the linear dynamics. To date, nonlinear resonances (in the sense of resonant driving of collective modes) have not been investigated in kicked systems.

Ensuring dynamical stability of the condensate is also very important in studies of its coherent dynamics: if the condensate is dynamically unstable, numbers of noncondensate particles grow exponentially. If it is stable, they grow more slowly (polynomially). More broadly, the study of different types of instability in static [15] and driven BECs [16] is of much current interest.

Previous work on kicked systems [3,6,7] considered the onset of dynamical instability and investigated the relation with classical chaos. In Ref. [3], the possibility that instability was related to chaos in the one-body limit was investigated for the kicked harmonic oscillator. In Refs. [6,7] the correlation between chaos in the mean-field dynamics, rather, and the onset of dynamical instability, was investigated. An "instability border," determined by the kick strength $K$ and the nonlinearity $g$ was mapped out; it was then found [7] that the parameter ranges for this border corresponds closely to a transition from regular to chaotic motion, of an effective classical Hamiltonian derived from the mean-field dynamics. Hence, present understanding of onset of dynamical instability in kicked BECs suggests that it may somehow be related to a transition to chaos.

In this work, we conclude that a quite different mechanism is primarily responsible for dynamical instability in the QKR-BEC. Our key finding is that it is the strong resonant driving of certain condensate modes by the kicking, which triggers loss of stability of the condensate. This mechanism is unrelated to the transition to chaos, but is rather an example of parametric resonance. In another context, the relationship between parametric resonance and dynamical instability of a BEC in a trap modulated periodically in time, is a topic of much current theoretical $[16,18]$ and experimental interest [19]. To date, "Bogoliubov spectroscopy" in the analogous time-periodic system, the $\delta$-kicked BEC, has never been investigated.

Our study shows that the temporally kicked BECs open up new possibilities in this arena. A key finding is that, for the rational values of kick period $T / \pi=m$ (where $m$ is integer), our model suggests that resonant excitation can involve two excited Bogoliubov modes. Hence we can explain the position of the critical stability border found in Refs. $[6,17]$. For irrational $T$, only single-mode Bogoliubov resonances have been identified to date. This sensitive dependence of the character of the Bogoliubov resonant excitation on whether the period is a rational or irrational multiple of $\pi$ is a specific feature of the kicked systems (as opposed to, for example, traps modulated periodically in time).

We find that, in general, for weak driving (small $K$ and $g$ ) the number of noncondensed atoms $N_{\mathrm{ex}}(t)$ grows exponentially only very close to a few, isolated resonance peaks. With increasing $K$ and $g$, the number of resonances which can be strongly excited by the kicking proliferates and overlaps. Our calculations show this is associated with generalized exponential instability; however, this regime is, to a large degree, beyond the scope of our methods. For moderate $K$ and $g$, though, we introduce a simple perturbative model which provides the approximate position and width of the 
important resonances for both rational and irrational $T$.

We investigate both the usual QKR-BEC as well as a simple modification, obtained by applying a series of pairs of closely spaced opposing kicks (the QKR2-BEC). This modifies substantially the relative strengths of the resonances, and provides the added novelty that the lowest modes are excited by an effective imaginary kick strength. It is closely related to the double-kicked quantum rotor, investigated in cold atoms experiments and theory [20]. We introduce a simple analytical model based on the properties of the unperturbed condensate, which gives the distinctive properties and scaling behavior of the condensate oscillations on and off resonance.

In Sec. II we introduce briefly the kicked and doublekicked BEC systems. In Sec. III we introduce the timedependent Bogoliubov method proposed by Castin and Dum and present numerics for the growth of noncondensate atoms. In Sec. IV we introduce a simple perturbative model, based on the one period time evolution operator for a kicked BEC. In Sec. V we show that the simple model and the time-dependent Bogoliubov numerics give excellent agreement in the limit of weak kicks. In Sec. VI we consider the case $T=2 \pi$ with both numerics and the perturbative model and show that the instability border found in Refs. [6,7] is due to a type of compound Bogoliubov resonance.

\section{KICKED BEC SYSTEMS}

As in Ref. [6], we consider a BEC confined in a ringshaped trap of radius $R$. We assume that the lateral dimension $r$ of the trap is much smaller than its circumference, and thus we are dealing with an effectively 1D system [21]. The dynamics of the condensate wave function at temperatures well below the transition temperature are then governed by the 1D Gross-Pitaevskii (GP) Hamiltonian with an additional kicking potential

$$
H=H_{\mathrm{GP}}+K \cos \theta f(t),
$$

yielding a dimensionless GP equation

$$
H_{\mathrm{GP}}=-\frac{1}{2} \frac{\partial^{2}}{\partial \theta^{2}}+g|\psi(\theta, t)|^{2}
$$

The short-range interactions between the atoms in the condensate are described by a mean-field term with scaled strength $g=8 N_{\text {tot }} a_{S} R / r^{2}$, where $a_{S}$ is the $s$-wave scattering length, and $N_{\text {tot }}$ is the total number of atoms. The length is measured in units of $R$ and energy in units of $\frac{\hbar^{2}}{2 m R^{2}}$ [6]. For the QKR-BEC system $f(t)=\Sigma_{n} \delta(t-n T)$, while for the QKR2BEC

$$
f(t)=\sum_{n}[\delta(t-n T)-\delta(t-n T+\epsilon)],
$$

where $T$ is the total period of the driving; $\epsilon \ll T$, and thus the second kick nearly cancels the first.

Experimental and theoretical studies of the double-kicked rotor [20] have shown that its quantum behavior is largely determined by an effective kick strength $K_{\epsilon}=K \epsilon$, provided $T \gg \epsilon$. Here we take $\epsilon=1 / 25$. Hence, while for the QKR-
BEC, the value $K=1$ represents a relatively large impulse for a kicked BEC, for a double kicked BEC, $K=1$ in the numerics below corresponds to $K_{\epsilon}=0.04$, and represents only a very weak impulse. The reason for this is the near cancellation of consecutive kicks in each pair.

This mechanism has certain analogies with the so-called "quantum antiresonance" investigated in Ref. [6]: for QKRs kicked at $T=2 \pi$, consecutive kicks effectively cancel. This means that even large values of $K \simeq 1$ and $g>1$ represent only weak driving; for example, the instability border was found by Ref. [6] to occur at $g \simeq 2$ and $K=0.8$.

\section{TIME-DEPENDENT BOGOLIUBOV METHOD}

The number of noncondensed atoms were calculated by making the usual Bogoliubov approximation, and following the formalism of Castin and Dum [22]. This adaptation of the Bogoliubov linearization for time-dependent potentials has been used in all studies to date of the dynamical stability of kicked condensates $[3,6,13,17]$. The mean number of noncondensed atoms at zero temperature is given by $N_{\text {ex }}(t)$ $=\sum_{k=1}^{\infty}\left\langle v_{k}(t) \mid v_{k}(t)\right\rangle$, where the amplitudes $\left(u_{k}, v_{k}\right)$ of the Bogoliubov quasiparticle operators are governed by the coupled equations

$$
i \hbar \frac{\partial}{\partial t}\left(\begin{array}{l}
u_{k} \\
v_{k}
\end{array}\right)=\left(\begin{array}{cc}
H+g Q|\psi|^{2} Q & g Q \psi^{2} Q^{*} \\
-g Q^{*} \psi^{* 2} Q & -H-g Q^{*}|\psi|^{2} Q^{*}
\end{array}\right)\left(\begin{array}{l}
u_{k} \\
v_{k}
\end{array}\right) .
$$

In this expression, $Q=I-|\psi\rangle\langle\psi|$ are projection operators that orthogonalize the quasiparticle modes with respect to the condensate [22]. We assume that at time $t=0$, we have a homogeneous condensate $\psi_{0}=1 / \sqrt{2 \pi}$. Further discussion of the theory is given in Ref. [23].

The regime of validity of the method is discussed in Ref. [22]. The method is valid in the weakly interacting limit 1 $\gg a_{s}^{3} \rho$, where $\rho$ is the density. A limit is identified where this condition is satisfied, if one works with a constant $g$ $\propto N_{\text {tot }} a_{s}$; thus the limit $a_{s} \rightarrow 0$ corresponds to $N_{\text {tot }} \rightarrow \infty$. A further requirement is that condensate depletion remains negligible. This condition fails after a few kicks in exponentially unstable regions. Here the method is employed only to identify the parameter range for the onset of instability. We cutoff our calculations for $N_{\text {ex }}>10^{2}-10^{3}$ (a reasonable threshold for small depletion in a condensate with $N_{\text {tot }} \sim 10^{5}$ ).

In Figs. 1(a) and 1(b) we show the number of noncondensed atoms $N_{\text {ex }}(t=N T)$, calculated from the Bogoliubov Eqs. (4) after $N=200$. For small $K=0.2, g=1$, a single resonance is seen at $T \simeq 10$. For small $K$, resonances occur whenever the resonance condition [16] $\omega_{l} \approx \frac{2 n \pi}{T}$ is satisfied, where $n=1,2,3, \ldots$, is an integer and $\omega_{l}$ is the eigenfrequency of the $l$ th collective mode. For larger $K=1$, the figure shows that resonances are extremely dense and overlap with each other (and we show the behavior in this regime for $T<10$ ). For overlapping resonances, unambiguous identification of each resonance is no longer possible. The key point here, however, is that in the stable regions outside the resonances, $N_{\text {ex }}$ remains very small even after prolonged kicking.

Figure 1(b) shows oscillations of $N_{\mathrm{ex}}$, as a function of time, for weak $K=0.2, g=1$, close to the isolated resonance 

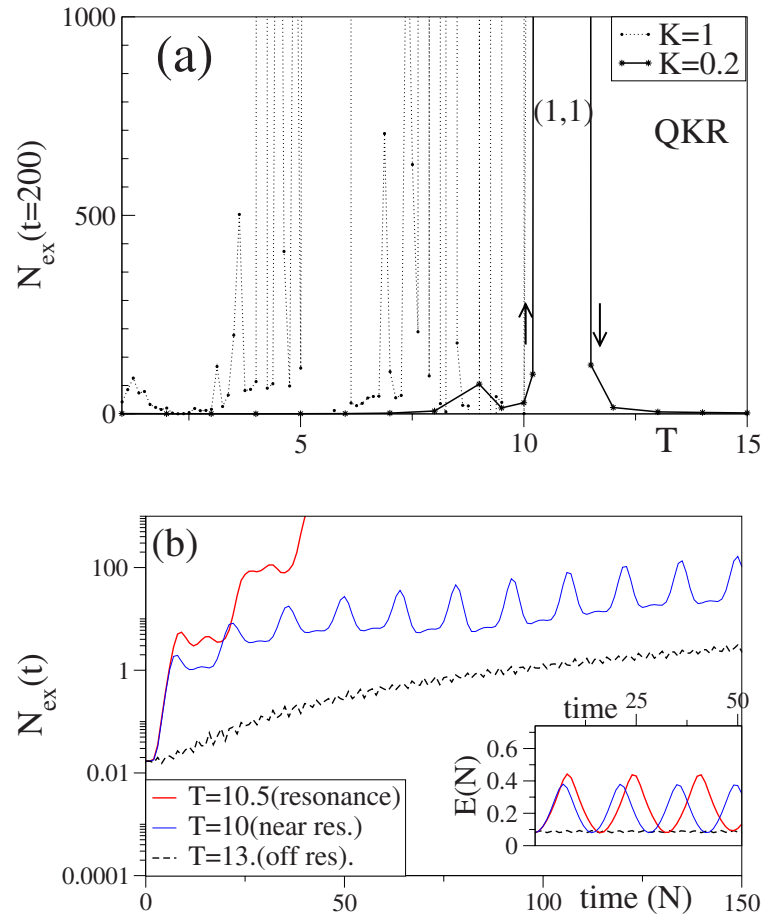

FIG. 1. (Color online) (a) Shows that for weak kicks (solid line), instability occurs only at one isolated Bogoliubov $(1,1)$ resonance, where $(n, l)$ denotes the $n$th resonance of eigenmode $l$. "Up" arrows indicate onset of exponential instability; " down" arrows means stability is regained. $g=1$. The total number of noncondensate atoms generated after 200 kicks, $N_{\mathrm{ex}}(N=200)$, is plotted as a function of kicking period $T$. For stronger kicks (dotted line; $K=1, T<10$ ) resonances proliferate and there is instability over almost all the parameter range. (b) Time dependence near the $(1,1)$ resonance at $T \approx 10$ corresponding to (a). Nonresonant $(T=13)$ curve shows weak quasiperiodic oscillations in $N_{\mathrm{ex}}$; the near-resonant regime, $T=10$ is characterized by slow, large oscillations; at resonance $T$ $=10.5$, there is exponential growth in $N_{\mathrm{ex}}(t)$. Inset shows that the condensate energy (calculated from the GPE itself) has similar oscillations.

at $T \approx 10$. The three possible regimes of (nonresonant) weak quasiperiodic oscillations in time; (near-resonant) slower, large periodic oscillations; and (resonant) exponential growth are illustrated. The condensate energy $E(N)=\int_{0}^{2 \pi} d \theta \psi^{*}(N)$ $\times\left[-\frac{1}{2} \frac{\partial^{2}}{\partial \theta^{2}}+\frac{g}{2}|\psi(N)|^{2}\right] \psi(N)$ after $N$ kicks, obtained from the GPE itself, is also shown, for comparison, in the inset: at resonance, large oscillations are also seen.

Figure 2 shows the corresponding behavior for the double-kicked BEC, but now as a function of $g$, keeping $T$ $=2, \epsilon=1 / 25$ constant, and $K=1$ or $K=5$ (hence $K_{\epsilon}=0.04$ or 0.2 ). The curve $K_{\epsilon}=0.04$ corresponds to weak impulses and shows two isolated Bogoliubov resonances.

While values of $g \simeq 1-10$ are large (but not unreasonably so) compared with current experimental values, resonances nearer small $g \sim 1$ more suitable for experimental spectroscopy can be identified by considering larger $T$ and other parameter ranges. For these effective one-dimensional systems, typical experimental values of $g_{1 \mathrm{D}}$ (in units of the recoil energy $E_{R}$ ) include $g \sim 0.5$ [13] (see discussion of experimental $g$ in Ref. [13]); or for a study of dynamics in a 1D

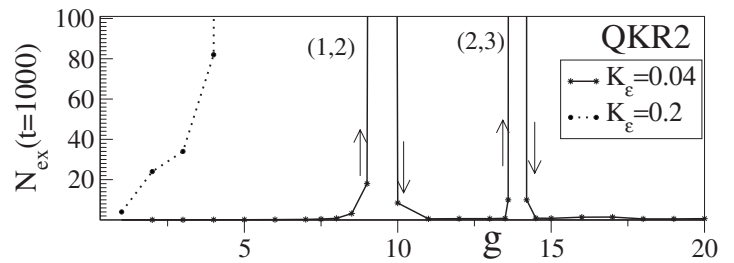

FIG. 2. Double-kicked BEC (QKR2): Shows zones of instability occur at Bogoliubov resonances. Condensate losses as a function of nonlinearity parameter $g$. "Up" arrows indicate onset of exponential instability; " down" arrows means stability is regained. $N_{\text {ex }}(t$ $=1000$ ) is plotted as a function of $g$ (for $T=2, \epsilon=1 / 25$ ) for weak kicks ( $K=1$ so effective kick is $\left.K_{\epsilon}=0.04\right)$ and stronger kicks $(K$ $=5$ so effective kick $K_{\epsilon}=0.2$ ).

modulated optical lattice a value $g_{1 \mathrm{D}}=0.73 E_{R}$, based on experiment, was considered [16]. Even larger values of $g$ are, in principle, attainable using Feshbach resonances or by varying the transverse confinement of a quasi-onedimensional condensate.

The curve $K_{\epsilon}=0.2$ is in the overlapping resonance regime, so produces generalized instability. In order to understand the behavior at the resonances, we now introduce a model for the time evolution of perturbations from the kicked condensate, based on the usual linearization with respect to small perturbations.

\section{KICKED CONDENSATE MODEL}

The time evolution of small perturbations of the condensate itself are described by an equation similar to Eq. (4), see Ref. [22]. We write the condensate wave function in the form $\psi=\psi_{0}+\delta \psi$, where $\psi_{0}$ is the unperturbed condensate and $\delta \psi$ represent the excited components. Inserting this form in the GPE and linearizing with respect to $\delta \psi$, we can write

$$
i \hbar \frac{\partial}{\partial t}\left(\begin{array}{c}
\delta \psi \\
\delta \psi^{*}
\end{array}\right)=\mathcal{L}(t)\left(\begin{array}{c}
\delta \psi \\
\delta \psi^{*}
\end{array}\right),
$$

where

$$
\mathcal{L}(t)=\left(\begin{array}{cc}
H(t)+g|\psi|^{2} & g \psi^{2} \\
-g \psi^{* 2} & -H(t)-g|\psi|^{2}
\end{array}\right) .
$$

The analysis of condensate stability for a time-periodic system [16] reduces to the analysis of the operator $\mathcal{L}(t)$ over one period $T$. In general, for systems similar to BECs in modulated optical lattices, intermode coupling requires a detailed analysis of the instantaneous evolution. The nature of the $\delta$-kicked potential permits considerable simplification.

The effect of $\mathcal{L}(t)$ reduces to the free-ringing of the eigenmodes of the unperturbed condensate for period $T$, interspersed by instantaneous impulses which mix the modes. Even for an experiment (where the kicks are approximated by pulses of very short, but finite duration) numerical time propagation is avoided: intermode coupling occurs over a very short time scale, during which eigenmode phases remain essentially constant.

Excluding the kick term for the moment, we recall that the time propagation under $H_{\mathrm{GP}}$ can be analyzed in terms of 
the eigenmodes $\left(u_{k}(t), v_{k}(t)\right)$ and eigenvalues of $\omega_{k}(t)$ of the $2 \times 2$ matrix on the right-hand side of Eq. (6). Setting $\psi$ $=1 / \sqrt{2 \pi}$, the matrix can be diagonalized and there are wellknown analytical expressions for the unperturbed eigenmodes [1]

$$
\left(u_{k}(t=0), v_{k}(t=0)\right)=\left(\begin{array}{c}
U_{k} \\
V_{k}
\end{array}\right) \frac{e^{i k \theta}}{\sqrt{2 \pi}},
$$

where $U_{k}+V_{k}=A_{k}, U_{k}-V_{k}=A_{k}^{-1}$ and $A_{k}=\left[\frac{\hbar^{2} k^{2}}{2}\left(\frac{\hbar^{2} k^{2}}{2}+\frac{g}{\pi}\right)\right]^{1 / 4}$.

In order to understand the behavior at the resonances, we introduce below a simple model using the eigenmodes Eq. (7) as a basis. Writing the small perturbation in this basis:

$$
\left(\begin{array}{c}
\delta \psi(t) \\
\delta \psi^{*}(t)
\end{array}\right)=\sum_{k} b_{k}(t)\left(\begin{array}{c}
U_{k} \\
V_{k}
\end{array}\right) \frac{e^{i k \theta}}{\sqrt{2 \pi}}+b_{k}^{*}(t)\left(\begin{array}{c}
V_{k} \\
U_{k}
\end{array}\right) \frac{e^{-i k \theta}}{\sqrt{2 \pi}} .
$$

Neglecting the kick, evolving the modes from some initial time $t_{0}$, each eigenmode $\left(u_{k}, v_{k}\right)$ simply acquires a phase, i.e.,

$$
b_{k}(t)=b_{k}\left(t_{0}\right) e^{-i \omega_{k}\left(t-t_{0}\right)} \text {, }
$$

where $\omega_{k}=\sqrt{\frac{k^{2}}{2}\left(\frac{\hbar^{2} k^{2}}{2}+\frac{g}{\pi}\right)}$.

After a time interval $T$, a kick is applied which couples the eigenmodes. Its effect is obtained by expressing the perturbation in a momentum basis $\psi=\Sigma_{l} a_{l}(t)|l\rangle$, where $|l\rangle=\frac{e^{i l \theta}}{\sqrt{2 \pi}}$, and we can restrict ourselves to the symmetric subspace $a_{l}$ $=a_{-l}$ of the initial condensate (parity is conserved in our system). Then, we can see by inspection that

$$
a_{k}(t)=U_{k} b_{k}(t)+V_{k} b_{-k}^{*}(t) .
$$

Note that $b_{k}=b_{-k}$ for this system. Conversely, the corresponding amplitude $b_{k}$ in each eigenmode $k$ is given trivially from Eq. (8) using orthonormality of the momentum states and the relation $U_{k}^{2}-V_{k}^{2}=1$, yielding

$$
b_{k}(t)=U_{k} a_{k}(t)-V_{k} a_{k}^{*}(t) .
$$

If the evolving condensate is given in the momentum basis, the effect of a kick operator $U_{\text {kick }}=e^{ \pm i(K / \hbar) \cos \theta}$ is well known. Its matrix elements are

$$
U_{n l}=\left\langle n\left|U_{\text {kick }}\right| l\right\rangle=J_{n-l}(K / \hbar) i^{ \pm(l-n)} .
$$

The $J_{n-l}$ are Bessel functions.

The amplitudes $a_{l}(t)$ are given by

$$
a_{n}\left(t^{+}\right)=\sum_{l} i^{ \pm(l-n)} J_{n-l}\left(\frac{K}{\hbar}\right) a_{l}\left(t^{-}\right),
$$

where $a_{n}\left(t^{+}\right) / a_{n}\left(t^{-}\right)$denotes the amplitude in state $|n\rangle$ just after/before the kick. We can now define a "time-evolution" operator $\mathcal{L}^{\prime}(T)=\mathcal{B}^{-1} \mathcal{L}_{\text {free }}(T) \mathcal{B} U_{\text {kick}}$, where $\mathcal{L}_{\text {free }}$ denotes free ringing of the eigenmodes, $\mathcal{B}$ is the transformation from momentum basis to Bogoliubov basis, and $U_{\text {kick }}$ is the action of the kick. A usual procedure [16] for stability analysis of a driven condensate is to examine the eigenvalues of an operator such as $\mathcal{L}^{\prime}(T)$ to ascertain whether they produce exponential growth in the amplitudes $a_{ \pm l}$.

However, to compare with GPE numerics, we simply evolve the mode amplitudes in time over a few kicks and examine the overall condensate response to the kicking (in

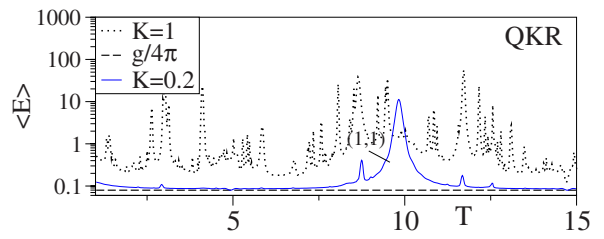

FIG. 3. (Color online) Average energy $\langle E\rangle$ after 40 kicks. The dashed lines indicate the model of Eq. (14); all other plots use full numerics. The label $(n, l)$ denotes $n$th resonance of mode $l$. Resonances of the QKR-BEC for parameters comparable to Fig. 1(a). For low $g=1, K=0.2$, only the single isolated $(1,1)$ resonance is seen. For higher $K=1$, resonances proliferate and overlap.

the limit of very weak kicking). Hence we can evolve the amplitudes $a_{l}(t=N T)$ of the condensate perturbation from period $N$ to period $N+1$ :

$$
\mathbf{a}((N+1) T)=\mathcal{L}^{\prime}(T) \mathbf{a}(N),
$$

using only the simple analytical coefficients in Eqs. (13) and (9), provided we use the simple transformations in Eqs. (10) and (11) to switch between the Bogoliubov mode basis and the momentum basis. $\mathcal{L}^{\prime}(T)$ is nonunitary, but the method is quantitative in the perturbative limit provided $\psi \simeq \psi_{0}$, i.e., if we assume $a_{0}(N) \simeq a_{0}(0) \simeq 1$.

We calculate the average energy over the first few $N$ kicks, $\langle E(N)\rangle=\frac{1}{N} \sum_{t=1}^{N} E(t)$. Slow, large amplitude oscillations in $E(t)$ yield a large $\langle E(t)\rangle$ and indicate a resonance. Figure 3 shows the QKR-BEC behavior, for equivalent parameters to Fig. 1(a). For low $K=0.2$, there is the same single $(1,1)$ resonance at $T \approx 10$ as in Fig. 1(a). For higher $K=1$ the method is far from quantitative: the model Eq. (14) is only a valid means of time evolving the perturbation over a few kicks for small $K \ll 1$ since it assumes the perturbed component is negligible; nevertheless, for $K=1$ it illustrates the regime of dense, overlapping resonances.

In Fig. 4 we compare the perturbative Eq. (14) results with full GPE numerics for the first 20 kick pairs of the QKR2 in the limit of weak kicks. It shows remarkably good agreement. Moreover the scaling of the resonances with $K$ is well described. The QKR2 resonant Bogoliubov spectrum differs appreciably from the QKR case. Figure 4 shows that

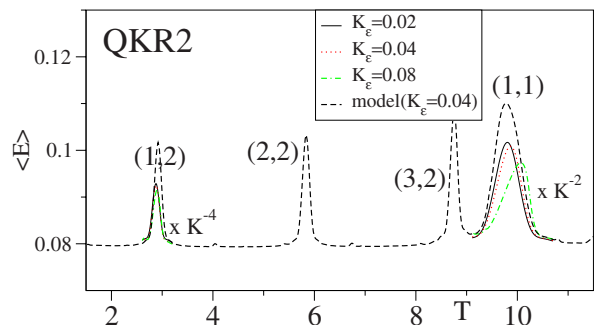

FIG. 4. (Color online) Comparison between full GPE numerics and the model of Eq. (14) for the QKR2-BEC, showing excellent agreement. Average energy $\langle E\rangle$ after 20 kick pairs. The label $(n, l)$ denotes $n$th resonance of mode $l . g=1$ and $\epsilon=1 / 25$ so $K=1$ corresponds to effective kick strength $K_{\epsilon}=0.04$. For low $K$, the $l=1$ resonance amplitudes scale as $\sim K^{2}$ while those of the $l=2$ modes scale as $\sim K^{4}$. 
for QKR2, even for low $K=1, \epsilon=1 / 25$, i.e., $K_{\epsilon} \approx 0.04$ and low $g=1$, both $l=1$ and $l=2$ resonances are strongly excited. The QKR2-BEC resonance intensity depends strongly on $K$ : the $l=2$ resonances scale as $K^{4}$, while the $l=1$ scaling is closer to $K^{2}$. In the full GPE numerics, the position of the maxima depends slightly on $K$ and $g$, but remains within a few percent of the unperturbed value, even for longer kicking times if $K_{\epsilon}$ remains small.

In the limit of weak driving, one can obtain explicit expressions for the condensate wave function as a function of time. We assume that $a_{0} \approx 1 / \sqrt{2} \pi \gg a_{l \neq 0}$. Then Eq. (13) can be approximated by $a_{l}\left(t^{+}\right) \approx a_{l}\left(t^{-}\right)+U_{l 0} /(\sqrt{2} \pi)$. From Eqs. (11) and (9) we see that the amplitude accumulated over a single period in each eigenmode is

$$
b_{l}(N+1)=\left[b_{l}(N)+\left(U_{l} U_{l 0}-V_{l} U_{l 0}^{*}\right)\right] e^{i \omega_{l} T} .
$$

Summing all contributions iteratively from $t=0$, taking $b_{l}(0)=0$, we obtain

$$
b_{l}(N)=\left(U_{l} U_{l 0}-V_{l} U_{l 0}^{*}\right) \sum_{n=0}^{N-1} e^{i n \omega_{l} T},
$$

and so for $\omega_{l} T \approx 2 \pi$ all the contributions add in phase, analogously to the well-known (but unrelated) resonances of the noninteracting limit [11].

We can write $\Sigma_{n=0}^{N-1} e^{i n \omega_{l} T}=e^{-i(N-1) \omega_{l} T} \Phi\left(\frac{N \omega_{l} T}{2}\right)$, where the $\Phi$ function is

$$
\Phi\left(\frac{N \omega_{l} T}{2}\right)=\frac{\sin \left(N \omega_{l} T / 2\right)}{\sin \left(\omega_{l} T / 2\right)} .
$$

We thus expect oscillations in each set of $\pm l$ momentum components of amplitude

$$
\left|2 a_{l}(N)\right|^{2} \propto 4\left|U_{l 0}\right|^{2} \Phi^{2}\left(\frac{N \omega_{l} T}{2}\right) .
$$

Off-resonance there will be quasiperiodic oscillations (in, e.g., the condensate energy) from the superposition of contributions characterized by different eigenfrequencies $\omega_{l}$. Close to resonance, a single component dominates; if the $l$ th mode is resonant we can write $\omega_{l} T \approx 2 \pi M+2 \delta$, where $2 \delta$ $\ll 1$ is the dephasing from resonance. Then

$$
\left|a_{l}(N)\right|^{2} \propto \frac{\left|U_{l 0}\right|^{2}}{\delta^{2}} \sin ^{2}(N \delta),
$$

and there are slow, periodic oscillations of large amplitude $\sim 4 \frac{\left|U_{0}\right|^{2}}{\delta^{2}}$, at a frequency $\delta$ which is not related to any eigenmode frequency, but given rather by the dephasing from resonance. The QKR2 resonant excitation spectrum is rather different from the QKR, and is analyzed further in the next section.

\section{RESONANCES OF THE QKR2-BEC}

In the limit $K_{\epsilon} \rightarrow 0$, we can obtain analytical expressions for the BEC wave function of the double-kicked system. First note that when $g \epsilon \ll 1$, the nonlinearity has little effect during the short time interval $\epsilon$. Using the relation

$$
e^{+i(K / \hbar) \cos \theta} e^{-i p^{2} t \hbar / 2} e^{-i(K / \hbar) \cos (\theta)}=e^{-(i t / 2 \hbar)[\hat{p}+K \sin \theta]^{2}},
$$

the time evolution can be given as a "one-kick" operator

$$
\hat{U}(T) \approx U_{\mathrm{GP}}^{(0)}(T, 0) e^{-(i \epsilon / 2 \hbar)[\hat{p}+K \sin \theta]^{2}} .
$$

In the limit $p \epsilon \approx 0$, one can split the operators in Eq. (21) and neglect a term $K \sin \theta \hat{p}$ to obtain the approximation

$$
\hat{U}(T) \approx e^{-(i / 2 \hbar) \hat{p}^{2} T} e^{-(i / \hbar)\left[\left(K^{2} \epsilon / 2\right) \sin ^{2} \theta-i K \epsilon \hbar \cos \theta\right]},
$$

leaving an effective single-kick quantum rotor with a kicking potential

$$
V_{\text {kick }}=\left[\frac{K^{2} \epsilon}{2} \sin ^{2} \theta-i K \epsilon \hbar \cos \theta\right] \sum_{N} \delta(t-N T) .
$$

The second term, curiously, appears as kicking potential with an imaginary, and $\hbar$ dependent, kick strength $i K \hbar$. It is of purely quantum origin as it arises from the noncommutativity of $p$ and $\sin \theta$, i.e.,

$$
i K \hbar \cos \theta=[K \sin \theta, \hat{p}] .
$$

Nevertheless, as seen below, it is important for weak driving as it controls the amplitude of the first excited mode $l= \pm 1$.

The matrix elements of the modified kick $V_{\text {kick}}$, similar to those in Eq. (13), are Bessel functions. Specifically, the effect of $V_{\text {kick }}$ on the condensate amplitudes $a_{l}$ is given by

$$
a_{n}\left(t^{+}\right)=\sum_{l} U_{n l} a_{l}\left(t^{-}\right)
$$

where $U_{n l}=\Sigma_{m} i^{n-l-m} J_{m}\left(\frac{K^{2} \epsilon}{4 \hbar}\right) J_{n-l-2 m}(i K \epsilon)$, and $a_{n}\left(t^{ \pm}\right)$indicates momentum amplitudes before(-) and after $(+)$ the kick, as in Eq. (13). Since $K \epsilon \ll 1$ and $J_{|n|>1}(z) \simeq 0$, only Bessel functions of low order ( $m=0$ or 1$)$ will be non-negligible, and we can use the small-argument approximations for them, namely, $J_{0}(z) \approx 1, J_{ \pm 1}(z) \approx \pm z / 2$.

Then, if the condensate is relatively unperturbed, the main effect of the kick will be to simply excite a small amount of $l= \pm 1$ and $l= \pm 2$ from the $|0\rangle$ state

$$
e^{-(i / \hbar) V_{\text {kick }} \psi \approx e^{-(i / \hbar)} V_{\text {kick }}|0\rangle}=\sum_{l} U_{l 0}|0\rangle,
$$

where

$$
\begin{aligned}
\sum_{l} U_{l 0}|0\rangle & \approx \frac{1}{\sqrt{2 \pi}}+i J_{1}\left(\frac{i K \epsilon}{2}\right)| \pm 1\rangle+i J_{1}\left(\frac{K^{2} \epsilon}{4 \hbar}\right)| \pm 2\rangle \\
& \approx \frac{1}{\sqrt{2 \pi}}-\frac{K \epsilon}{4}| \pm 1\rangle+i \frac{K^{2} \epsilon}{8 \hbar}| \pm 2\rangle
\end{aligned}
$$

We obtain a similar equation to the QKR-BEC for the mode amplitudes, i.e.,

$$
b_{l}(N)=\left(U_{l} U_{l 0}-V_{l} U_{l 0}^{*}\right) \sum_{n=0}^{N-1} \exp \left[i n \omega_{l} T\right] .
$$

However, if only the lowest excited modes are significant, then, in particular, 

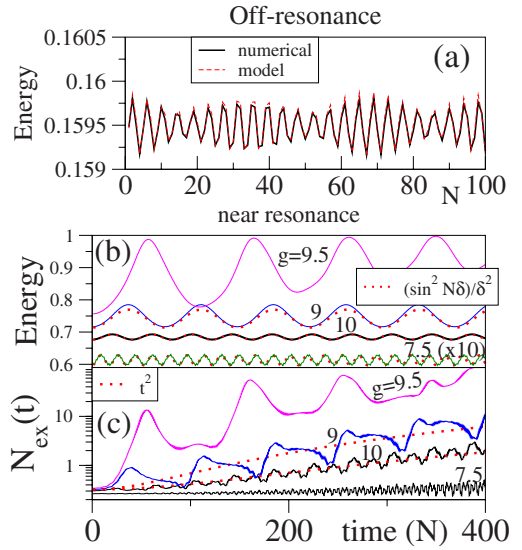

FIG. 5. (Color online) Test of perturbative model. (a) Condensate energy oscillations from GPE numerics and Eq. (28). $K_{\epsilon}$ $=0.04, g=2, T=2$. Beating between modes 1 and 2 is very accurately described by Eq. (28). (b) Behavior of $l=2$ resonance of Fig. 1 (b) $K_{\epsilon}=0.04, T=2$, and $g \approx 9.5$. As the resonance is approached the amplitude of the oscillations is proportional to the square of their wavelength, i.e., $E(t=N T) \propto K^{4} \frac{1}{(\delta)^{2}} \sin ^{2} N \delta$, where $2 \delta$ is the distance from the resonance peak. (c) Corresponding number of noncondensate atoms from Eq. (4).

$$
b_{1}(N)=-\frac{K \epsilon}{4}\left(U_{1}-V_{1}\right) \sum_{n=0}^{N-1} \exp \left[i n \omega_{1} T\right]
$$

and $b_{2}(N)=i \frac{K^{2} \epsilon}{8 \hbar}\left(U_{2}+V_{2}\right) \sum_{n=0}^{N-1} \exp \left[i n \omega_{2} T\right]$. For $\omega_{l} T \approx 2 \pi$ all the contributions add in phase and we will have a resonance of either the $l=1$ or $l=2$ modes, the regime illustrated in Fig. 2(b).

Similarly as for the QKR-BEC, we can sum all the contributions to obtain an approximate analytical expression for the evolving condensate wave function including excited modes $l= \pm 1$ and $l= \pm 2$,

$$
\psi(N) \approx \frac{1}{2 \pi}\left[1+C_{1} \frac{K \epsilon}{2} \cos \theta+C_{2} \frac{K^{2} \epsilon}{4 \hbar} \cos 2 \theta\right],
$$

where

$$
\begin{gathered}
C_{1}=-\Phi\left(N \widetilde{\omega}_{1}\right)\left[\cos (N-1) \tilde{\omega}_{1}-i A_{1}^{-2} \sin (N-1) \tilde{\omega}_{1}\right], \\
C_{2}=\Phi\left(N \widetilde{\omega}_{2}\right)\left[A_{2}^{2} \sin (N-1) \tilde{\omega}_{2}+i \cos (N-1) \widetilde{\omega}_{2}\right],
\end{gathered}
$$

and $\widetilde{\omega}_{j}=\omega_{j} T / 2$.

Equation (28) shows that the amplitudes $\left|a_{1}\right|^{2}$ and $\left|a_{2}\right|^{2}$ scale as $K^{2}$ and $K^{4}$, respectively, as seen in the numerics in Fig. 4. Figure 5(a) shows that Eq. (28) gives excellent agreement with GPE numerics, giving accurately the nonresonant quasiperiodic condensate oscillations. Near the $l=2$ resonance of Fig. 2, Fig. 5(b) confirms the QKR2 condensate oscillations (obtained from the GPE) scale quite accurately as $\propto \frac{1}{(\delta)^{2}} \sin ^{2} N \delta$ as expected from Eqs. (19) and (28).

Figure 5(c) shows that, near resonance, there are corresponding large oscillations in the noncondensate numbers calculated from Eq. (4). Near-resonance $N_{\text {ex }}$ increases quadratically with time, on-resonance, the increase is exponential.
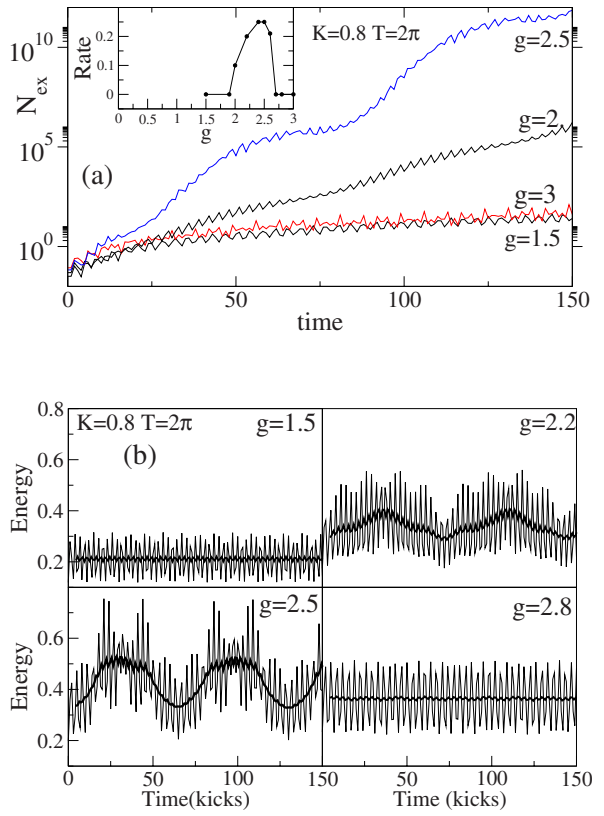

FIG. 6. (Color online) (a) Noncondensate particles for kicking period $T=2 \pi, K=0.8, g \simeq 2$. The inset shows the rate of exponential growth of noncondensate atoms; zero denotes polynomial growth or less. The graph shows this instability border is a resonance: the condensate is unstable for $g=2-2.5$ but is stable for $g=1.5$ and $g$ =3.0. (b) Energy oscillations as a function of time; smoothed plots are also shown. Before and after the resonance $(g=1.5$ and $g=2.8)$ the smoothed plots are flat. Near resonance $(g=2.2$ and $g=2.5)$ the energy shows the characteristic slow, deep resonant oscillations.

\section{BOGOLIUBOV RESONANCES FOR $T=\mathbf{2} \pi$}

The kick period $T=2 \pi$, in a noninteracting system of cold atoms (i.e., $g=0$ ) corresponds to a so-called "quantum antiresonance," where the cold atom cloud exhibits periodic (period-2) oscillations. Hence the isolated Bogoliubov resonance regime persists to higher $K$ than would be expected for generic $T$. The effect of a nonzero $g$ for $T=2 \pi$ was investigated in Ref. [6]. An instability border occurring at a critical value of nonlinearity, e.g., for $g \simeq 2$ at $K=0.8$, was identified where the growth on noncondensate particles with time became exponential.

In Fig. 6(a) we investigate the behavior near critical $g$, for $K=0.8$. We see that if a wider range of $g$ is considered, the stability border is also a resonance: the condensate rapidly recovers stability after the instability border is passed. The condensate is exponentially unstable for $g \simeq 2 \rightarrow 2.6$, but is quite stable for both $g=1.5$ and $g=3$, as shown. Figure 6(b) shows oscillations in the condensate energy, as a function of time; a smoothed plot is also shown. For $g=1.5$ and $g=2.8$ (off resonance) the smoothed plots are flat; for $g=2.2$ and $g=2.5$ (near resonance), slow deep oscillations are apparent.

The behavior is analogous to that of generic $T$; however, the analysis of the condensate resonances for $T=2 \pi$ is less straightforward: the strongest resonances, even for low $K$ $\lesssim 2$, do not, in fact, occur for $\omega_{l} T \approx 2 \pi M$, where $M$ $=1,2,3, \ldots$. A significant difference between generic $T$ and $T=2 \pi$ is that, for the generic case, if we write 


$$
\omega_{l} T \approx 2 \pi M_{l}+2 \delta(l)
$$

we see that for arbitrary generic $T$, the distance from the nearest resonance, for the different modes, depends on $l$. In contrast, for $T=2 \pi$, for large $l$ (i.e., $l \gtrsim 3)$ we find $\omega_{l} T \approx\left(l^{2}\right.$ $\left.+\frac{g}{\pi}\right) \pi$; in other words, the dephasing from the nearest resonance (and hence the period of the mode oscillations) is similar (either $2 \delta \approx \frac{g}{\pi}$ or $2 \delta \approx 1-\frac{g}{\pi}$ ) for all modes. So all mode oscillations for high $l$ are approximately in phase with each other.

For $K=0.8$, only low modes $l=1,2$ are significantly populated. These low modes $(l=1$ and $l=2)$ are only in phase with each other at certain precise values of $g, T$. For these parameters, the model of Eq. (14) predicts large resonances whenever the condition $\left(\omega_{1}+\omega_{2}\right) T \approx 2 \pi M$ is satisfied. In particular, for the resonance near $g \approx 2$, we find that for the $l=1$ mode, $\omega_{1} T \approx(1-2 \delta) 2 \pi$ while for the $l=2$ mode $\omega_{2} T \approx(2$ $+2 \delta) 2 \pi$, with $2 \delta \approx 0.25$.

These results suggest that "two-mode resonances," i.e., synchronized oscillations of pairs of the lowest excited modes are the dominant mechanism for $T=2 \pi$. They account for the shifting position of the critical instability border found by Ref. [6] in the $T=2 \pi$ case. For example, for slightly higher kick strengths, such as $K \simeq 2$, a resonance appears for $g \approx 1.65$ corresponding to $\left(\omega_{2}+\omega_{3}\right) T \approx 2 \pi M$, which accounts for the displacement of the instability border to lower values of $g$. Note that the resonance positions in the full numerics are $K$ dependent, whereas in the perturbative model of Eq. (14) this dependence is neglected; the model is only valid for very small $K$.

\section{CONCLUSION}

In conclusion, we have shown that exponential instability in kicked BECs is related to parametric resonances, i.e., driving of low-lying collective modes at their natural frequencies, rather than to chaos in the underlying mean-field dynamics [24]. The signature of this process is in the onset of slow, large amplitude periodic oscillations in the condensate energy as well as the number of noncondensate atoms calculated from the time-dependent Bogoliubov formalism, as a resonance is approached. The resonances proliferate and overlap for large kick strengths $K$, leading to instability over wider ranges of $K$ and $g$. The time-dependent Bogoliubov approximation used here and in all other previous studies is only valid in regimes where the condensate depletion is negligible; for realistic condensates analysis of the dynamics in the narrow (for weak driving) windows of parametric instability, would require other approaches. However, away from these windows, the kicked condensate remains stable and relatively unperturbed, even after prolonged kicking.

\section{ACKNOWLEDGMENTS}

J.R. acknowledges funding from EPSRC-DHPA. The authors would like to thank Simon Gardiner, Mark Raizen, and Chuanwei Zhang for valuable advice. This research was supported by the EPSRC.
[1] L. P. Pitaevskii and S. Stringari, Bose-Einstein Condensation (Oxford University Press, Oxford, 2003); F. Dalfovo, S. Giorgini, L. P. Pitaevskii, S. Stringari, Rev. Mod. Phys. 71, 463 (1999).

[2] D. L. Shepelyansky, Phys. Rev. Lett. 70, 1787 (1993).

[3] S. A. Gardiner, D. Jaksch, R. Dum, J. I. Cirac, and P. Zoller, Phys. Rev. A 62, 023612 (2000); R. Artuso and L. Rebuzzini, Phys. Rev. E 68, 036221 (2003).

[4] Q. Thommen, J. C. Garreau, and V. Zehnle, Phys. Rev. Lett. 91, 210405 (2003).

[5] G.J. Duffy, A.S. Mellish, K.J. Challis, and A.C. Wilson, Phys. Rev. A 70, 041602(R) (2004).

[6] C. Zhang, J. Liu, M. G. Raizen, and Q. Niu, Phys. Rev. Lett. 92, 054101 (2004).

[7] J. Liu, C. Zhang, M.G. Raizen, and Q. Niu, Phys. Rev. A 73, 013601 (2006).

[8] S. Wimberger, R. Mannella, O. Morsch, and E. Arimondo, Phys. Rev. Lett. 94, 130404 (2005)

[9] A. D. Martin, C. S. Adams, and S. A. Gardiner, Phys. Rev. Lett. 98, 020402 (2007).

[10] M. G. Raizen, Adv. At., Mol., Opt. Phys. 41, 43 (1999).

[11] M. K. Oberthaler, R. M. Godun, M. B. d'Arcy, G. S. Summy, and K. Burnett, Phys. Rev. Lett. 83, 4447 (1999).

[12] S. Fishman, I. Guarneri, and L. Rebuzzini, Phys. Rev. Lett. 89, 084101 (2002); L. Rebuzzini, S. Wimberger, and R. Artuso, Phys. Rev. E 71, 036220 (2005).
[13] L. Rebuzzini, R. Artuso, S. Fishman, and I. Guarneri, Phys. Rev. A 76, 031603(R) (2007).

[14] C. Ryu, M. F. Andersen, A. Vaziri, M. B. d'Arcy, J. M. Grossman, K. Helmerson, and W. D. Phillips, Phys. Rev. Lett. 96, 160403 (2006); M. Sadgrove, M. Horikoshi, T. Sekimura, and K. Nakagawa, ibid. 99, 043002 (2007); G. Behinaein, V. Ramareddy, P. Ahmadi, and G. S. Summy, ibid. 97, 244101 (2006); I. Dana, V. Ramareddy, I. Talukdar, and G. S. Summy, ibid. 100, 024103 (2008); J. F. Kanem, S. Maneshi, M. Partlow, M. Spanner, and A. M. Steinberg, ibid. 98, 083004 (2007).

[15] B. Wu and Q. Niu, New J. Phys. 5, 104 (2003).

[16] M. Kramer, C. Tozzo, and F. Dalfovo, Phys. Rev. A 71, 061602(R) (2005); C. Tozzo, M. Krämer, and F. Dalfovo, ibid. 72, 023613 (2005).

[17] D. Poletti, G. Benenti, G. Casati, and B. Li, Phys. Rev. A 76, 023421 (2007)

[18] J. J. Garcia-Ripoll, V. M. Perez-Garcia, and P. Torres, Phys. Rev. Lett. 83, 1715 (1999); Yu. Kagan and L. A. Manakova, e-print arXiv:cond-mat/0609159.

[19] N. Gemelke, E. Sarajlic, Y. Bidel, S. Hong, and S. Chu, Phys. Rev. Lett. 95, 170404 (2005); G. Campbell, J. Mun, M. Boyd, E. Streed, W. Ketterle, and D. Pritchard, ibid. 96, 020406 (2006); P. Engels, C. Atherton, and M. A. Hoefer, ibid. 98, 095301 (2007).

[20] P. H. Jones, M. M. Stocklin, G. Hur, and T. S. Monteiro, Phys. 
Rev. Lett. 93, 223002 (2004); C. E. Creffield, G. Hur, and T. S. Monteiro, ibid. 96, 024103 (2006); J. Wang, T. S. Monteiro, S. Fishman, J. P. Keating, and R. Schubert, ibid. 99, 234101 (2007).

[21] This means that we work with the effective value of the onedimensional nonlinearity constant $g \equiv g_{1 \mathrm{D}}$.

[22] Y. Castin and R. Dum, Phys. Rev. Lett. 79, 3553 (1997); Y. Castin and R. Dum, 57, 3008 (1998).
[23] S.A.Gardiner and S.A.Morgan, Phys. Rev. A 75, 043621 (2007).

[24] While we cannot draw any conclusions on the kicked harmonic oscillator, as we do not study it, we note that, e.g., Figs. 18 and 19 in the study in Ref. [3] show deep slow oscillations suggestive of an approach to a Bogoliubov resonance (not necessarily leading to exponential behavior in those examples). 